\title{
EQUIDISTRIBUTION RESULTS FOR GEODESIC FLOWS
}

\author{
ABDELHAMID AMROUN \\ Université Paris Sud, Département de Mathématiques, CNRS UMR \\ 8628, 91405 Orsay Cedex France

\begin{abstract}
Using the works of Mañé [14 and Paternain [18] we study the distribution of geodesic arcs with respect to equilibrium states of the geodesic flow on a closed manifold, equipped with a $\mathcal{C}^{\infty}$ Riemannian metric. We prove large deviations lower and upper bounds and a contraction principle for the geodesic flow in the space of probability measures of the unit tangent bundle. We deduce a way of approximating equilibrium states for continuous potentials.
\end{abstract}

\section{INTRODUCTION}

Let $M$ be a closed and connected manifold equipped with a $C^{\infty}$ Riemannian metric. We study the distribution of geodesic $\operatorname{arcs}$ of $M$ with respect to equilibrium states. We prove large deviations lower and upper bounds for the geodesic flow in the space of probability measures of the unit tangent bundle. More precisely, we consider Lebesgue measures supported on a finite number of geodesic arcs and show that they define a process which satisfy a large deviation principle with action function given by the topological pressure. As an application, we obtain equidistribution results which describe the proportion of geodesic arcs which support Lebesgue measures close to equilibrium states. We show that this proportion converges exponentially fast to one when the length of the geodesic arcs tends to infinity. We also prove a contraction principle for these probability measures, which is a large deviation theorem with constraints. This work is based on two remarkable formulas due to Mañé [14] and Paternain [18] which characterize the topological entropy and pressure as a growth rate of the number of the geodesic $\operatorname{arcs}$ (see Theorem 8 and Theorem 9). On the other hand, the technics of convex analysis and large deviations in [11] and [9] were particulary usefull for this work. We adapt and extend certain of the general arguments in [1] to our situation. 
We have two important situations where the results of the paper apply. The first situation concerns the class of manifolds with negative curvature. In this case, it is well known that for any Hölder continuous potential there exists a unique equilibrium state. There are three well known invariant measures in this setting. The Bowen-Margulis measure, which is the equilibrium state (a measure of maximal entropy) corresponding to constant potentials. The harmonic measure which corresponds to the potential $\left.\frac{d}{d t}\right|_{t=0}\left(K \circ \widetilde{\varphi}^{t}\right)$ where $K$ is the Poisson kernel and $\widetilde{\varphi}^{t}$ the geodesic flow of $S \widetilde{M}$, where $\widetilde{M}$ is the universal cover of the manifold $M$. The Liouville measure which is the equilibrium state of the potential $\left.\frac{d}{d t}\right|_{t=0} \operatorname{det}\left(\left.d \varphi_{t}\right|_{E^{s}}\right)$ where $E^{s}$ is the stable tangent bundle of $S M$ (see [7] and [8] for more details). For the "Liouville potential", we obtain that the geodesic arcs are uniformly distributed with respect to the Liouville measure.

The second situation deals with the more general class of Riemannian manifolds of nonpositive curvature which are Rank 1 . Then by a result of Knieper [12] we know that there exists a uniquely determined invariant measure of maximal entropy for the geodesic flow. But it is not known up to now which class of raisonable potentials admit a unique equilibrium state and this question remains open for Rank 1 manifolds. However, our results give a way of approximating equilibrium states of the geodesic flow of such manifolds. Indeed, these results are applicable everywhere where Mañé's and Paternain's formulas hold.

Finally, I'm very grateful to François Ledrappier for helpful conversations.

\section{MAin RESUlts}

2.1. Preliminaries and notations. Let $M$ be a closed and connected manifold equipped with a $C^{\infty}$ Riemannian metric and $\phi: S M \rightarrow S M$ be the geodesic flow on the unit tangent bundle $S M$. We assume that $M$ has volume one, $\int_{M} d x=1$, where $d x$ is the volume form induced by the Riemannian metric of $M$.

We denote by $\mathcal{P}(S M)$ the space of probability measures on $S M$ equipped with the weak star topology. Let $\mathcal{P}_{\text {inv }}(S M)$ be the subset of $\mathcal{P}(S M)$ of invariant probability measures. Given a potential $F \in$ $C_{\mathbb{R}}(S M)$, the topological pressure of $F$ is the number defined by the variational principle [25],

$$
P(F)=\sup _{m \in \mathcal{P}_{\text {inv }}(S M)}\left(h(m)+\int_{S M} F d m\right),
$$


where $h(m)$ is the entropy of $m$. For $F=0$ this reduces to

$$
P(0)=\sup _{m \in \mathcal{P}_{\text {inv }}(S M)} h(m):=h_{\text {top }}
$$

where $h_{\text {top }}$ is the topological entropy of the geodesic flow.

An equilibrium state for $F$, is a measure $m \in \mathcal{P}_{\text {inv }}(S M)$ which achieves the maximum in (1),

$$
h(m)+\int_{S M} F d m=P(F) .
$$

We denote by $\mathcal{P}_{e}(F)$ the subset of $\mathcal{P}_{\text {inv }}(S M)$ of equilibrium states corresponding to $F$. By a result of Newhouse [16, since the metric is $C^{\infty}$, the entropy map $m \rightarrow h(m)$ is upper semicontinuous. Then $h_{t o p}<\infty$ and consequently, the set $\mathcal{P}_{e}(F)$ is a nonempty closed, compact, convex subset of $\mathcal{P}(S M)$ [25].

We define the functional $Q_{F}$ on $C_{\mathbb{R}}(S M)$ based on the potential $F$ by,

$$
Q_{F}(\omega):=P(F+\omega)-P(F) .
$$

By definition, $Q_{F}$ is continuous on continuous functions (see Lemma 1 and Remark 1). Sometimes we will simply write $Q$, if there is no confusion to be been afraid.

We set for any probability measure $\mu$ on $S M$,

$$
J_{F}(\mu):=\sup _{\omega}\left(\int \omega d \mu-Q_{F}(\omega)\right),
$$

where the sup is taken over the space of continuous functions $\omega$ on $S M$. Observe that since $Q_{F}(0)=0$, then $J_{F}$ is a non negative functional and clearly is lower semicontinuous. We will see that (Lemma 1 and Remark 1 ) that $\mathcal{P}_{e}(F)=\left\{J_{F}=0\right\}$. Again, if there is no ambiguity we write $J$ instead of $J_{F}$.

By duality, we have

$$
Q_{F}(\omega)=\sup _{\mu \in \mathcal{P}(S M)}\left(\int \omega d \mu-J_{F}(\mu)\right) .
$$

For any set $E \subset \mathcal{P}(S M)$ put

$$
J_{F}(E):=\inf _{\mu \in E} J_{F}(\mu) .
$$

Given $x$ and $y$ in $M$, we denote by $\gamma_{x y}:\left[0, l\left(\gamma_{x y}\right)\right] \rightarrow M$ a unit speed geodesic arc joining $x$ to $y$ with length $l\left(\gamma_{x y}\right)$. For any $\delta>0$ and $T>0$ we set,

$$
\begin{aligned}
G_{\delta, T}(x, y) & :=\left\{\gamma_{x y}: T-\delta<l\left(\gamma_{x y}\right) \leq T\right\} \text { and } \\
G_{T}(x, y) & :=\left\{\gamma_{x y}: l\left(\gamma_{x y}\right) \leq T\right\}
\end{aligned}
$$


Recall from [3] that given $T>0$, the set of geodesic arcs with length $\leq T$ is finite and its cardinality is locally constant for an open full Lebesgue measure subset of $M \times M$ (see also [18] p53 for a proof using Sard's theorem). By a result of Burns K and Gutkin E [4], the growth of $\# G_{T}(x, y)$ and a positive topological entropy are related to the condition of "insecurity" of the manifold.

The integral $\int_{\gamma_{x y}} \omega$ of a function $\omega$ over the geodesic arc $\gamma_{x y}$ is defined by

$$
\int_{\gamma_{x y}} \omega:=\int_{0}^{l\left(\gamma_{x y}\right)} \omega\left(\phi_{s}\left(\dot{\gamma}_{x y}(0)\right) d s .\right.
$$

Here $\dot{\gamma}_{x y}(0)$ is the initial condition of the geodesic $\gamma_{x y}$. The Lebesgue measure $\delta_{\gamma_{x y}}$ with support in $\gamma_{x y}$ is now defined by,

$$
\int_{S M} \omega d \delta_{\gamma_{x y}}:=\frac{1}{l\left(\gamma_{x y}\right)} \int_{\gamma_{x y}} \omega
$$

We will write $G_{\delta, T}$ and $G_{T}$ for simplicity, since the dependence in $(x, y)$ will be always clear.

2.2. The results. Consider a real continuous potential $F$ defined on $S M$. For any Borel subset $E$ of $\mathcal{P}(S M)$ we set,

$$
\nu_{T}(E):=\frac{\int_{M \times M}\left(\sum_{\gamma_{x y} \in G_{\delta, T}: \delta_{\gamma_{x y}} \in E} e^{\int_{\gamma_{x y}} F}\right) d x d y}{\int_{M \times M}\left(\sum_{\gamma_{x y} \in G_{\delta, T}} e^{\int_{\gamma_{x y}} F}\right) d x d y} .
$$

This defines a process $\left(\nu_{T}\right)_{T>0}$ on the space $\mathcal{P}(S M)$. The first result gives large deviations bounds for this process, namely

$$
\begin{aligned}
& \limsup _{T \rightarrow+\infty} \frac{1}{T} \log \nu_{T}(K) \leq-J(K) \\
& \liminf _{T \rightarrow+\infty} \frac{1}{T} \log \nu_{T}(O) \geq-J(O),
\end{aligned}
$$

respectively, for any closed subset $K$ and open subset $O$ of $\mathcal{P}(S M)$.

Before we state the main theorem, we do the following assumption under which we prove the lower bound part of the large deviation theorem. This condition is well known when we deal with the lower bound part (see [11] and [9]).

There exists a countable set $\mathcal{C}:=\left\{g_{k}, k \geq 1\right\} \subset C_{\mathbb{R}}(S M)$ of continuous fucntions such that their span is dense in $C_{\mathbb{R}}(S M)$ with respect to the topology of uniforme convergence, $\left\|g_{k}\right\|=1$ for all $k$, and for all $\beta \in \mathbb{R}^{n}$ the potential $\sum_{k=1}^{n} \beta_{k} g_{k}$ has a unique equilibrium state. 
Theorem 1. Let $M$ be a closed and connected manifold equipped with a $C^{\infty}$ Riemannian metric and $F \in C_{\mathbb{R}}(S M)$. Then for any $\delta>0$ we have

(1) For any closed subset $K$ of $\mathcal{P}(S M)$

$$
\limsup _{T \rightarrow+\infty} \frac{1}{T} \log \frac{\int\left(\sum_{\gamma_{x y} \in G_{\delta, T}: \delta_{\gamma_{x y}} \in K} e^{\int_{\gamma_{x y}} F}\right) d x d y}{\int\left(\sum_{\gamma_{x y} \in G_{\delta, T}} e^{\int_{\gamma_{x y}} F}\right) d x d y} \leq-J(K) .
$$

(2) If for all $\beta \in \mathbb{R}^{n}$ and $g=\left(g_{1}, \ldots, g_{n}\right) \in \mathcal{C}^{n}, F+\beta \cdot g$ has a unique equilibrium state, then for any open subset $O$ of $\mathcal{P}(S M)$,

$$
\liminf _{T \rightarrow+\infty} \frac{1}{T} \log \frac{\int\left(\sum_{\gamma_{x y} \in G_{\delta, T}: \delta_{\gamma_{x y}} \in O} e^{\int_{\gamma_{x y}} F}\right) d x d y}{\int\left(\sum_{\gamma_{x y} \in G_{\delta, T}} e^{\int_{\gamma_{x y}} F}\right) d x d y} \geq-J(O) .
$$

As consequence of Theorem 1 (1) we have the following result which asserts that the proportion of geodesic arcs supporting a Lebesgue measure close to an equilibrium state is asymptotically equal to 1 : $\lim _{T \rightarrow \infty} \nu_{T}(V)=1$ and the convergence is exponential. Set $V^{c}:=$ $\mathcal{P}(S M) \backslash V$.

Corollary 1. For any open neighborhood $V$ of $\mathcal{P}_{e}(F)$ in $\mathcal{P}(S M)$ we have

$$
\lim _{T \rightarrow+\infty} \frac{\int\left(\sum_{\gamma_{x y} \in G_{\delta, T}: \delta_{\gamma_{x y}} \in V} e^{\int_{\gamma_{x y}} F}\right) d x d y}{\int\left(\sum_{\gamma_{x y} \in G_{\delta, T}} e^{\int_{\gamma_{x y}} F}\right) d x d y}=1,
$$

where the convergence is exponential with speed $e^{-T J\left(V^{c}\right)}$.

We know that large deviation principles are preserved under continuous mapping, this is known as the contraction principle ([9]). In the present case, the contraction principle reduces the preceeding theorem to a finite dimensional one. First, we need some notations.

Set for $g \in C_{\mathbb{R}^{n}}(S M)$ and $\alpha \in \mathbb{R}^{n}, n>0$,

$$
\mathcal{P}_{g, \alpha}(S M):=\left\{m \in \mathcal{P}(S M): \int_{S M} g d m=\alpha\right\} .
$$

We define the functional

$$
J_{g}(\alpha)=\left\{\begin{array}{lll}
\inf \left(J(m): m \in \mathcal{P}_{g, \alpha}(S M)\right. & \text { if } & \mathcal{P}_{g, \alpha}(S M) \neq \emptyset \\
+\infty & \text { if } & \mathcal{P}_{g, \alpha}(S M)=\emptyset
\end{array}\right.
$$

and for any $E_{n} \subset \mathbb{R}^{n}$,

$$
J_{g}\left(E_{n}\right)=\inf \left(J_{g}(\alpha): \alpha \in E_{n}\right) .
$$

To simplify the notations set $m(g)=\int g d m$ for $m \in \mathcal{P}(S M)$. 
Theorem 2 (Contraction principle). Let $M$ be a closed and connected manifold equipped with a $C^{\infty}$ Riemannian metric and $F \in C_{\mathbb{R}}(S M)$. Let $g \in C_{\mathbb{R}^{n}}(S M)$. Then for any $\delta>0$ we have

(1) For any closed subset $K_{n} \subset \mathbb{R}^{n}$,

$$
\limsup _{T \rightarrow+\infty} \frac{1}{T} \log \frac{\int\left(\sum_{\gamma_{x y} \in G_{\delta, T}: \delta_{\gamma_{x y}}(g) \in K_{n}} e^{\int_{\gamma_{x y}} F}\right) d x d y}{\int\left(\sum_{\gamma_{x y} \in G_{\delta, T}} e^{\int_{\gamma_{x y}} F}\right) d x d y} \leq-J_{g}\left(K_{n}\right) .
$$

(2) If for all $\beta \in \mathbb{R}^{n}, F+\beta \cdot g$ has a unique equilibrium state, then for any open subset $O_{n} \subset \mathbb{R}^{n}$,

$$
\liminf _{T \rightarrow+\infty} \frac{1}{T} \log \frac{\int\left(\sum_{\gamma_{x y} \in G_{\delta, T}: \delta_{\gamma_{x y}}(g) \in O_{n}} e^{\int_{\gamma_{x y}} F}\right) d x d y}{\int\left(\sum_{\gamma_{x y} \in G_{\delta, T}} e^{\int_{\gamma_{x y}} F}\right) d x d y} \geq-J_{g}\left(O_{n}\right) .
$$

Part (1) of Theorem 2 is a consequence of Theorem 1 (1), by the continuity of the function $g$. Note that we do not assume in part (2) of Theorem 2 that $g \in \mathcal{C}^{n}$. This last condition is used in the proof of part (2) of Theorem 1. In other words, the conclusion of Theorem 2 (2) holds for any continuous $g$ such that for all $\beta \in \mathbb{R}^{n}, F+\beta \cdot g$ admits a unique equilibrium state.

For any $\delta>0$ we define the probability measures on $S M$,

$$
m_{\delta, T}:=\frac{\int\left(\sum_{\gamma_{x y} \in G_{\delta, T}} e^{\int_{\gamma_{x y}} F} \cdot \delta_{\gamma_{x y}}\right) d x d y}{\int\left(\sum_{\gamma_{x y} \in G_{\delta, T}} e^{\int_{\gamma_{x y}} F}\right) d x d y} .
$$

As an application of Theorem 1 (1) we prove the following theorem.

Theorem 3. Let $M$ be a closed and connected manifold equipped with $a C^{\infty}$ Riemannian metric and $F \in C_{\mathbb{R}}(S M)$. For any $\delta>0$, any weak limit $m_{\infty}$ of $\left(m_{\delta, T}\right)_{T}$ is an equilibrium states for the potential $F$, i.e $m_{\infty}$ is invariant and satisfies

$$
h\left(m_{\infty}\right)+\int F d m_{\infty}=P(F) .
$$

These theorems apply to the geodesic flow of a manifold of negative curvature and Hölder continuous potentials $F$. The following result is particulary important when the unique equilibrium state $\mu_{F}$ corresponding to $F$ is the Bowen-Margulis measure or the harmonic measure (section 1). In particular, if $F$ is the "Liouvile potential" (section 1) then this corollary says that the geodesic arcs are uniformly distributed.

Corollary 2. Let $M$ be a closed and connected manifold of negative curvature equipped with a $C^{\infty}$ Riemannian metric. Suppose that the 
potential $F$ is Hölder continuous and let $\mu_{F}$ be the unique corresponding equilibrium state. Then, for any $\delta>0$, the measures $\left(m_{\delta, T}\right)_{T}$ converge weakly to $\mu_{F}$ as $T \rightarrow+\infty$.

Consider now the measure $\mu_{\max }$ of maximal entropy [12] of the geodesic flow of a rank 1 manifold. Set

$$
\mu_{\delta, T}:=\frac{\int\left(\sum_{\gamma_{x y} \in G_{\delta, T}} \delta_{\gamma_{x y}}\right) d x d y}{\int\left(\sum_{\gamma_{x y} \in G_{\delta, T}}\right) d x d y}
$$

Corollary 3. Let $M$ be a closed and connected rank 1 manifold equipped with a $C^{\infty}$ Riemannian metric. Then, for any $\delta>0$, the measures $\left(\mu_{\delta, T}\right)_{T}$ converge weakly to $\mu_{\max }$ as $T \rightarrow+\infty$.

\section{Constant And Positive potentials}

We state in this section some results which are not a direct consequence of the previous ones.

3.1. Positive potentials. Consider the probability measures defined on $S M$ by

$$
m_{T}:=\frac{\int\left(\sum_{\gamma_{x y} \in G_{T}} e^{\int_{\gamma_{x y}} F} \delta_{\gamma_{x y}}\right) d x d y}{\int\left(\sum_{\gamma_{x y} \in G_{T}} e^{\int_{\gamma_{x y}} F}\right) d x d y}
$$

Theorem 4. Let $M$ be a closed and connected manifold equipped with a $C^{\infty}$ Riemannian metric and $F \in C_{\mathbb{R}}(S M)$. Assume that $P(F)>0$. Then, the weak limits of $\left(m_{T}\right)_{T}$ are equilibrium states corresponding to the potential $F$. If $M$ has negative curvature then $\left(m_{T}\right)_{T}$ converges to the unique equilibrium state $\mu_{F}$ corresponding to $F$.

Theorem 4 is a consequence of part 1 of the following theorem.

Theorem 5. Let $M$ be a closed and connected manifold equipped with a $C^{\infty}$ Riemannian metric and $F \in C_{\mathbb{R}}(S M)$. Then

(1) If $P(F)>0$ we have for any closed subset $K$ of $\mathcal{P}(S M)$ such that $J(K)<P(F)$,

$$
\limsup _{T \rightarrow+\infty} \frac{1}{T} \log \frac{\int\left(\sum_{\gamma_{x y} \in G_{T}: \delta_{\gamma_{x y} \in K}} e^{\int_{\gamma_{x y}} F}\right) d x d y}{\int\left(\sum_{\gamma_{x y} \in G_{T}} e^{\int_{\gamma_{x y}} F}\right) d x d y} \leq-J(K) .
$$


(2) If for all $\beta \in \mathbb{R}^{n}$ and $g=\left(g_{1}, \ldots, g_{n}\right) \in \mathcal{C}^{n}, F+\beta \cdot g$ has a unique equilibrium state, then for any open subset $O$ of $\mathcal{P}(S M)$,

$$
\liminf _{T \rightarrow+\infty} \frac{1}{T} \log \frac{\int\left(\sum_{\gamma_{x y} \in G_{T}: \delta_{\gamma_{x y}} \in O} e^{\int_{\gamma_{x y}} F}\right) d x d y}{\int\left(\sum_{\gamma_{x y} \in G_{T}} e^{\int_{\gamma_{x y}} F}\right) d x d y} \geq-J(O) .
$$

Observe that given $F$ and any constant $c$ we have $\mathcal{P}_{e}(F)=\mathcal{P}_{e}(F+c)$. On the other hand, we can find a constant $c>0$ such that $F+c>0$. Thus up to a constant we can always assume that $P(F)>0$.

3.2. Constant potentials. We consider here constant potentials which is equivalent to set $F \equiv 0$.

Theorem 6. Suppose that $M$ is a closed and connected manifold equipped with a $C^{\infty}$ Riemannian metric. If $M$ has no conjugate points, then for any $\delta>0$,

(1) For any closed subset $K$ of $\mathcal{P}(S M)$ and a.e $(x, y) \in M \times M$

$$
\limsup _{T \rightarrow+\infty} \frac{1}{T} \log \frac{\#\left\{\gamma_{x y} \in G_{\delta, T}: \delta_{\gamma_{x y}} \in K\right\}}{\# G_{\delta, T}} \leq-J(K) .
$$

(2) For a.e $(x, y) \in M \times M$, the weak limits of

$$
\mu_{\delta, T}(x, y):=\frac{\sum_{\gamma_{x y} \in G_{\delta, T}} \delta_{\gamma_{x y}}}{\# G_{\delta, T}}
$$

are measures of maximal entropy.

In part (1) of Theorem 6 , the set of points $(x, y) \in M \times M$ for which we have the upper bound depends on the given closed set $K$, while in part (2) it depends only on $\delta$.

Theorem 7. Suppose that $M$ is a closed and connected manifold equipped with a $C^{\infty}$ Riemannian metric. If $M$ has no conjugate points, and $h_{\text {top }}>0$ then,

(1) For any closed subset $K$ of $\mathcal{P}(S M)$ such that $J(K)<h_{\text {top }}$ and a.e $(x, y) \in M \times M$

$$
\limsup _{T \rightarrow+\infty} \frac{1}{T} \log \frac{\#\left\{\gamma_{x y} \in G_{T}: \delta_{\gamma_{x y}} \in K\right\}}{\# G_{T}} \leq-J(K) .
$$

(2) For a.e $(x, y) \in M \times M$, the weak limits of

$$
\mu_{T}(x, y):=\frac{\sum_{\gamma_{x y} \in G_{T}} \delta_{\gamma_{x y}}}{\# G_{T}}
$$

are measures of maximal entropy. 


\section{Proofs}

4.1. Growth of geodesic arcs. The following two theorems of Mañé [14] and Paternain [18] are the main tools in the proof of our results.

Theorem 8 (R Mañé [14]). Let $M$ be a closed and connected manifold equipped with a $C^{\infty}$ metric. Then

$$
h_{\text {top }}=\lim _{T \rightarrow \infty} \frac{1}{T} \log \int_{M \times M} \# G_{T}(x, y) d x d y .
$$

(2) If $M$ has no conjugate points, for all $(x, y)$

$$
h_{\text {top }}=\lim _{T \rightarrow \infty} \frac{1}{T} \log \# G_{T}(x, y) .
$$

(3) Suppose that the metric is of class $C^{3}$ and $M$ does not have conjugate points. Then for any $\delta>0$ and all $(x, y)$ we have

$$
h_{\text {top }}=\lim _{T \rightarrow \infty} \frac{1}{T} \log \# G_{\delta, T}(x, y) .
$$

Theorem 9 (G P Paternain [18]). Let $M$ be a closed and connected manifold equipped with a $C^{\infty}$ metric.

(1) For any $\delta>0$

$$
P(F)=\lim _{T \rightarrow+\infty} \frac{1}{T} \log \int_{M \times M}\left(\sum_{\gamma_{x y} \in G_{\delta, T}} e^{\int_{\gamma_{x y}} F}\right) d x d y .
$$

(2) If $P(F) \geq 0$

$$
P(F)=\lim _{T \rightarrow+\infty} \frac{1}{T} \log \int_{M \times M}\left(\sum_{\gamma_{x y} \in G_{T}} e^{\int_{\gamma_{x y}} F}\right) d x d y .
$$

For any invariant probability measure $\mu$ we set

$$
I(\mu):=P(F)-\left(h(\mu)+\int_{S M} F d \mu\right) .
$$

Lemma 1. (1) The functional $Q_{F}$ is convex and continuous on continuous functions.

(2) $Q(\omega)=\sup _{\mu \in \mathcal{P}_{\text {inv }}(S M)}\left(\int \omega d \mu-I(\mu)\right)$. In other words, the functionals $I$ and $J_{F}$ agree on invariant measures.

Proof. Part (1) is a consequence of the convexity of the pressure function $P$ and the variational principle (1) from which we can easily deduce 
that $|P(f)-P(g)| \leq\|f-g\|_{\infty}$ [25]. Part (2) follows from (7) and,

$$
\begin{aligned}
& \sup _{\mu \in \mathcal{P}_{\text {inv }}(S M)}\left(\int \omega d \mu-I(\mu)\right) \\
= & \sup _{\mu \in \mathcal{P}_{\text {inv }}(S M)}\left(\int \omega d \mu-P(F)+h(\mu)+\int F d \mu\right) \\
= & P(F+\omega)-P(F)=Q_{F}(\omega) .
\end{aligned}
$$

Remark 1. $Q_{F}$ is invariant by the geodesic flow : $Q_{F}\left(\omega \circ \phi_{t}\right)=Q_{F}(\omega)$, for all $t$ and continuous function $\omega$. Thus, a probability measure $m$ satisfies $J_{F}(m)=0$ if and only if $m$ is invariant and $I(m)=0$. In particular, if $K$ is a closed subset of $\mathcal{P}(S M)$ and $\inf _{m \in K} J_{F}(m)=$ $J_{F}(\mu)$ for some $\mu \in K$, then $\inf _{m \in K} J_{F}(m)=0$ iff $\mu$ is invariant and $h(\mu)+\int F d \mu=P(F)$. In other words we have $\mathcal{P}_{e}(F)=\left\{J_{F}=0\right\}$.

\subsection{Proof of Theorem 1 (1).}

Proof. We have to prove

$$
\limsup _{T \rightarrow \infty} \frac{1}{T} \log \nu_{T}(K) \leq-\inf _{m \in K} J(m):=-J(K) .
$$

Let $\epsilon>0$. There exists a finite number of continuous functions $\omega_{1}, \cdots, \omega_{l}$ such that $K \subset \cup_{i=1}^{l} K_{i}$, where

$$
K_{i}=\left\{m \in \mathcal{P}(S M): \int \omega_{i} d m-Q\left(\omega_{i}\right)>J(K)-\epsilon\right\} .
$$

Put

$$
\Gamma_{i}(x, y, T):=\left\{\gamma_{x y} \in G_{\delta, T}: \delta_{\gamma_{x y}} \in K_{i}\right\}
$$

and

$$
Z_{i}(T):=\int_{M \times M} \sum_{\gamma_{x y} \in \Gamma_{i}(x, y, T)} e^{\int_{\gamma_{x y}} F} d x d y .
$$

We have $\nu_{T}(K) \leq \sum_{i=1}^{l} \nu_{T}\left(K_{i}\right)$ and

$$
\nu_{T}\left(K_{i}\right)=\frac{Z_{i}(T)}{\int_{M \times M}\left(\sum_{\gamma_{x y} \in G_{\delta, T}} e^{\int_{\gamma_{x y}} F}\right) d x d y} .
$$

We have

$$
Z_{i}(T) \leq \int_{M \times M} \sum_{\gamma_{x y} \in \Gamma_{i}(x, y, T)} e^{\int_{\gamma_{x y}} F} e^{l\left(\gamma_{x y}\right)\left(\int \omega_{i} d \delta_{\gamma_{x y}}-Q\left(\omega_{i}\right)-(J(K)-\epsilon)\right)} d x d y
$$


Set $C:=\sum_{i \leq l} \sup \left(1, e^{-\delta\left(-Q\left(\omega_{i}\right)-(J(K)-\epsilon)\right)}\right)$. Thus, by taking into account the sign of $-Q\left(\omega_{i}\right)-(J(K)-\epsilon)$,

$$
\begin{aligned}
Z_{i}(T) & \leq \int_{M \times M} \sum_{\gamma_{x y} \in \Gamma_{i}(x, y, T)} e^{\int_{\gamma_{x y}}\left(F+\omega_{i}\right)} e^{l\left(\gamma_{x y}\right)\left(-Q\left(\omega_{i}\right)-(J(K)-\epsilon)\right)} d x d y \\
& \leq C e^{T\left(-Q\left(\omega_{i}\right)-(J(K)-\epsilon)\right)} \int_{M \times M} \sum_{\gamma_{x y} \in \Gamma_{i}(x, y, T)} e^{\int_{\gamma_{x y}}\left(F+\omega_{i}\right)} d x d y
\end{aligned}
$$

For $T$ sufficiently large, it follows from Theorem 9 (1),

$$
\begin{aligned}
\nu_{T}(K) & \leq \sum_{i=1}^{l} \frac{Z_{i}(T)}{\int \sum_{\gamma_{x y} \in G_{\delta, T}} e^{\int_{\gamma_{x y}} F} d x d y} \\
& \leq C \sum_{i=1}^{l} e^{T\left(P\left(F+\omega_{i}\right)+\epsilon\right)} e^{-T(P(F)-\epsilon)} e^{T\left(-Q\left(\omega_{i}\right)-(J(K)-\epsilon)\right.} \\
& =C l e^{T(-J(K)+3 \epsilon)} .
\end{aligned}
$$

Take the logarithme, divide by $T$ and the limsup,

$$
\limsup _{T \rightarrow \infty} \frac{1}{T} \log \nu_{T}(K) \leq-J(K)+3 \epsilon .
$$

$\epsilon$ being arbitrary, this proves Theorem 1 (1).

\subsection{Proof of Corollary 1.}

Proof. It suffices to apply Theorem 1 to the closed set $K=\mathcal{P}(S M) \backslash V$. We have $J(K)=J(m)$ for some $m \in K$ and $\nu_{T}(V)=1-\nu_{T}(K)$. By Remark $1, J(m)>0$ and for $T$ sufficiently large,

$$
1 \geq \nu_{T}(V) \geq 1-e^{T J(m)} .
$$

\subsection{Proof of Theorem 2 (2).}

Proof. Recall that $\mathcal{P}_{g, \alpha}(S M)=\left\{m \in \mathcal{P}(S M): \int g d m=\alpha\right\}$ and

$$
\begin{gathered}
J_{g}(\alpha)=\inf \left(J(m): m \in \mathcal{P}_{g, \alpha}(S M)\right) \\
J_{g}\left(O_{n}\right):=\inf _{\alpha \in O_{n}} J_{g}(\alpha) .
\end{gathered}
$$

Given a continuous function $g: S M \rightarrow \mathbb{R}^{n}$ we set $\beta \cdot g=\sum_{i=1}^{n} \beta i g_{i}$ for $\beta \in \mathbb{R}^{n}$. Then, by definition of the function $Q$, we have

$$
Q(\beta \cdot g)=\sup _{\alpha \in \mathbb{R}^{n}}\left(\beta \cdot \alpha-J_{g}(\alpha)\right),
$$


and by duality,

$$
J_{g}(\alpha)=\sup _{\beta \in \mathbb{R}^{n}}(\beta \cdot \alpha-Q(\beta \cdot g)) .
$$

If $J_{g}\left(O_{n}\right)=+\infty$ then there is nothing to do. Suppose then $J\left(O_{n}\right)<$ $+\infty$. Let $\varepsilon>0$ and choose $\alpha_{\varepsilon} \in O_{n}$ with $\mathcal{P}_{g, \alpha_{\varepsilon}}(S M) \neq \emptyset$ such that

$$
J_{g}\left(O_{n}\right)>J_{g}\left(\alpha_{\varepsilon}\right)-\varepsilon \text {. }
$$

We know from ([23] Theorem 23.4 and 23.5) that, given $\alpha$ in the interior of the affine hull of the domain $D\left(J_{g}\right)$ of $J_{g}$, there exists $\beta \in \mathbb{R}^{n}$ such that

$$
Q(\beta \cdot g)=\beta \cdot \alpha-J_{g}(\alpha) .
$$

Let then $\beta_{\varepsilon} \in \mathbb{R}^{n}$ such that

$$
Q\left(\beta_{\varepsilon} \cdot g\right)=\beta_{\varepsilon} \cdot \alpha_{\varepsilon}-J_{g}\left(\alpha_{\varepsilon}\right) .
$$

Consider now a small neighborhood of $\alpha_{\varepsilon}$,

$$
O_{n, r}:=\left\{\alpha \in \mathbb{R}^{n}:\left|\alpha_{\varepsilon}-\alpha\right| \leq r\right\},
$$

such that $O_{n, r} \subset O_{n}$. Define for any $E \subset \mathbb{R}^{n}$ :

$$
\begin{aligned}
\Gamma_{T}(E) & :=\left\{\gamma_{x y} \in G_{\delta, T}: \delta_{\gamma_{x y}}(g) \in E\right\} \\
Z_{T}(E) & :=\frac{\int\left(\sum_{\gamma_{x y} \in \Gamma_{T}(E)} e^{\int_{\gamma_{x y}} F}\right) d x d y}{\int\left(\sum_{\gamma_{x y} \in G_{\delta, T}} e^{\int_{\gamma_{x y}} F}\right) d x d y} .
\end{aligned}
$$

We have, $Z_{T}\left(O_{n}\right) \geq Z_{T}\left(O_{n, r}\right)$ and

$$
\begin{aligned}
& \sum_{\gamma_{x y} \in \Gamma_{T}\left(O_{n, r}\right)} e^{\int_{\gamma_{x y}} F} \\
= & e^{-T \beta_{\varepsilon} \cdot \alpha_{\varepsilon}} \sum_{\gamma_{x y} \in \Gamma_{T}\left(O_{n, r}\right)} e^{\int_{\gamma_{x y}} F} e^{-T\left(\beta_{\varepsilon} \cdot\left(\delta_{\gamma_{x y}}(g)-\alpha_{\varepsilon}\right)\right)} e^{T \beta_{\varepsilon} \cdot \delta_{\gamma_{x y}}(g)} \\
\geq & e^{-T \beta_{\varepsilon} \cdot \alpha_{\varepsilon}} e^{-r\left\|\beta_{\varepsilon}\right\| T} \sum_{\gamma_{x y} \in \Gamma_{T}\left(O_{n, r}\right)} e^{\int_{\gamma_{x y}} F} e^{T \beta_{\varepsilon} \cdot \delta_{\gamma_{x y}}(g)} \\
= & e^{-T \beta_{\varepsilon} \cdot \alpha_{\varepsilon}} e^{-r\left\|\beta_{\varepsilon}\right\| T} \sum_{\gamma_{x y} \in \Gamma_{T}\left(O_{n, r}\right)} e^{\int_{\gamma_{x y}} F} e^{l\left(\gamma_{x y}\right) \beta_{\varepsilon} \cdot \delta_{\gamma_{x y}}(g)} e^{\left(T-l\left(\gamma_{x y}\right)\right) \beta_{\varepsilon} \cdot \delta_{\gamma_{x y}}(g)} .
\end{aligned}
$$

By the condition, $0 \leq T-l\left(\gamma_{x y}\right) \leq \delta$, we have

$$
e^{\left(T-l\left(\gamma_{x y}\right)\right) \beta_{\varepsilon} \cdot \delta_{\gamma_{x y}}(g)} \geq e^{-\delta\left\|\beta_{\varepsilon} \cdot g\right\|_{\infty}} .
$$

Thus

$$
\sum_{\gamma_{x y} \in \Gamma_{T}\left(O_{n, r}\right)} e^{\int_{\gamma_{x y}} F} \geq e^{-T \beta_{\varepsilon} \cdot \alpha_{\varepsilon}} e^{-r\left\|\beta_{\varepsilon}\right\| T} e^{-\delta\left\|\beta_{\varepsilon} \cdot g\right\|_{\infty}} \sum_{\gamma_{x y} \in \Gamma_{T}\left(O_{n, r}\right)} e^{\int_{\gamma_{x y}}\left(F+\beta_{\varepsilon} \cdot g\right)} .
$$


Set

$$
Z_{T}^{\varepsilon}\left(O_{n, r}\right):=\frac{\int\left(\sum_{\gamma_{x y} \in \Gamma_{T}\left(O_{n, r}\right)} e^{\int_{\gamma_{x y}}\left(F+\beta_{\varepsilon} \cdot g\right)}\right) d x d y}{\int\left(\sum_{\gamma_{x y} \in G_{\delta, T}} e^{\int_{\gamma_{x y}}\left(F+\beta_{\varepsilon} \cdot g\right)}\right) d x d y}
$$

and

$$
Z_{T}\left(\beta_{\varepsilon} \cdot g\right):=\frac{1}{T} \log \frac{\int\left(\sum_{\gamma_{x y} \in G_{\delta, T}} e^{\int_{\gamma_{x y}}\left(F+\beta_{\varepsilon} \cdot g\right)}\right) d x d y}{\int \sum_{\gamma_{x y} \in G_{\delta, T}} e^{\int_{\gamma_{x y}} F} d x d y}
$$

Therefore,

$\frac{1}{T} \log Z_{T}\left(O_{n, r}\right) \geq-r\left\|\beta_{\varepsilon}\right\|-\frac{\delta\left\|\beta_{\varepsilon} \cdot g\right\|_{\infty}}{T}+\left(Z_{T}\left(\beta_{\varepsilon} \cdot g\right)-\beta_{\varepsilon} \cdot \alpha_{\varepsilon}\right)+\frac{1}{T} \log Z_{T}^{\varepsilon}\left(O_{n, r}\right)$,

From Theorem 9 (1) and by definition of $Q=Q_{F}$ (see (2)) we get,

$$
\lim _{T \rightarrow \infty} Z_{T}\left(\beta_{\varepsilon} \cdot g\right)=P\left(F+\beta_{\varepsilon} \cdot g\right)-P(F)=Q\left(\beta_{\varepsilon} \cdot g\right) .
$$

Thus

$\liminf _{T \rightarrow \infty} \frac{1}{T} \log Z_{T}\left(O_{n}\right) \geq-r\left\|\beta_{\varepsilon}\right\|+\left(Q\left(\beta_{\varepsilon} \cdot g\right)-\beta_{\varepsilon} \cdot \alpha_{\varepsilon}\right)+\lim _{T \rightarrow \infty} \frac{1}{T} \log Z_{T}^{\varepsilon}\left(O_{n, r}\right)$.

We will show that

$$
\lim _{T \rightarrow \infty} Z_{T}^{\varepsilon}\left(O_{n, r}\right)=1
$$

Let us see how to finish the proof using (14) :

$$
\begin{aligned}
\liminf _{T \rightarrow \infty} \frac{1}{T} \log Z_{T}\left(O_{n}\right) & \geq-r\left\|\beta_{\varepsilon}\right\|+Q\left(\beta_{\varepsilon} \cdot g\right)-\beta_{\varepsilon} \cdot \alpha_{\varepsilon} \\
& =-r\left\|\beta_{\varepsilon}\right\|-J_{g}\left(\alpha_{\varepsilon}\right) \\
& \geq-r\left\|\beta_{\varepsilon}\right\|-J_{g}\left(O_{n}\right)-\varepsilon,
\end{aligned}
$$

for any $\varepsilon>0$. Since $r>0$ was arbitray choosen, we let $r \rightarrow 0$ and $\varepsilon \rightarrow 0$ respectively and we get $\liminf _{T \rightarrow \infty} \frac{1}{T} \log Z_{T}\left(O_{n}\right) \geq-J_{g}\left(O_{n}\right)$ which completes the proof Theorem 2 (2).

It remains to show (14). Let $K_{n, r}$ be the complement set of $O_{n, r}$ in the image $g *(\mathcal{P}(S M))$ of $\mathcal{P}(S M)$ under the continuous map $g *: m \rightarrow g \cdot m$. We have $Z_{T}^{\varepsilon}\left(O_{n, r}\right)+Z_{T}^{\varepsilon}\left(K_{n, r}\right)=1$. The goal is to show that $Z_{T}^{\varepsilon}\left(K_{n, r}\right)$ decrease exponentially fast to zero as $T \rightarrow \infty$ using Theorem 1 .

Consider $J^{\varepsilon}:=J_{F+\beta_{\varepsilon} \cdot g}$ which is the functional $J$ corresponding to $Q^{\varepsilon}:=Q_{F+\beta_{\varepsilon} \cdot g}$. We have

$$
Q^{\varepsilon}(\omega)=P\left(F+\beta_{\varepsilon} \cdot g+\omega\right)-P\left(F+\beta_{\varepsilon} \cdot g\right),
$$

and by (3),

$$
J^{\varepsilon}(m)=\sup _{\omega}\left(\int \omega d m-Q^{\varepsilon}(\omega)\right) .
$$


From this we deduce easily that

$$
J^{\varepsilon}(m)=J(m)+Q\left(\beta_{\varepsilon} \cdot g\right)-\int \beta_{\varepsilon} \cdot g d m
$$

and

$$
\inf _{m(g)=\alpha} J^{\varepsilon}(m)=\inf _{m(g)=\alpha} J(m)+Q\left(\beta_{\varepsilon} \cdot g\right)-\beta_{\varepsilon} \cdot \alpha .
$$

The set $K_{n, r}$ is compact in $\mathbb{R}^{n}$ and by Theorem 1 (1),

$$
\limsup _{T \rightarrow \infty} \frac{1}{T} \log Z_{T}^{\varepsilon}\left(K_{n, r}\right) \leq-J^{\epsilon}(K),
$$

with $K:=(g *)^{-1}\left(K_{n, r}\right)$ which is a closed subset of $\mathcal{P}(S M)$. We have,

$$
J^{\varepsilon}(K)=\inf _{\alpha \in K_{n, r}}\left(J_{g}(\alpha)+Q\left(\beta_{\varepsilon} \cdot g\right)-\beta_{\varepsilon} \cdot \alpha\right) .
$$

If $J^{\varepsilon}(K)=+\infty$ there is nothing to do and the result follows. The key point is to prove that $J^{\varepsilon}(K)>0$. Set

$$
J_{g}^{\varepsilon}(\alpha):=J_{g}(\alpha)+Q\left(\beta_{\varepsilon} \cdot g\right)-\beta_{\varepsilon} \cdot \alpha .
$$

The functional $J^{\varepsilon}$ is non negative (since $Q^{\varepsilon}(0)=0$ ), lower semicontinuous and then it achieves its minimum on compact sets. We have $J_{g}^{\varepsilon}(\alpha) \geq 0$ and $J_{g}^{\varepsilon}\left(\alpha_{\varepsilon}\right)=0$ (see (12)). Recall that, if $J_{g}^{\varepsilon}(\alpha)=0$ for some $\alpha \in K_{n, r}$, then there will correspond to $\alpha$ an equilibrium state $m_{\alpha} \in K$ for the potential $F+\beta_{\varepsilon} \cdot g$ such that $m_{\alpha}(g)=\alpha$. The vector $\alpha_{\varepsilon}$ is the unique point realizing the minimum, i.e the unique solution for the equation $J_{g}^{\varepsilon}(\alpha)=0$. Indeed, two different solutions will produce two distinct equilibrium states for the potential $F+\beta_{\varepsilon} \cdot g$ which contradicts our standing assumption of Theorem 1. Since $\alpha_{\varepsilon} \in O_{n, r}$, then $J_{g}^{\varepsilon}(\alpha)>0$ for $\alpha \in K_{n, r}$. On the other hand the set $K_{n, r}$ being compact, by the lower semicontinuity of $J^{\varepsilon}$ we have $J^{\varepsilon}(K)=\inf _{\alpha \in K_{n, r}} J_{g}^{\varepsilon}(\alpha)>0$. Thus we have proved that

$$
\limsup _{T \rightarrow \infty} \frac{1}{T} \log Z_{T}^{\varepsilon}\left(K_{n, r}\right) \leq-J^{\varepsilon}(K)<0
$$

from which (14) follows immediately.

\subsection{Proof of Theorem 1 (2).}

Proof. Let $O \subset \mathcal{P}(S M)$ be an open set and $\epsilon>0$. Choose $m_{\epsilon} \in O$ such that

$$
J\left(m_{\epsilon}\right) \leq J(O)+\epsilon
$$


We endow the space $\mathcal{P}(S M)$ with a compatible topology generated by the distance given by

$$
d\left(m, m^{\prime}\right):=\sum_{k=1}^{\infty} 2^{-k}\left|m\left(g_{k}\right)-m^{\prime}\left(g_{k}\right)\right|,
$$

where the functions $g_{k}$ were defined in section 2.2. Following [11] we define,

$$
d_{n}\left(m, m^{\prime}\right):=\sum_{k=1}^{n} 2^{-k}\left|m\left(g_{k}\right)-m^{\prime}\left(g_{k}\right)\right| .
$$

Set $2 r=\inf \left\{d\left(m, m_{\epsilon}\right): m \in \mathcal{P}(S M) \backslash O\right\}$. We have $r>0$, since $\mathcal{P}(S M) \backslash O$ is a compact subset of $\mathcal{P}(S M)$. Since for all $k,\left\|g_{k}\right\|=1$, we have $0 \leq d\left(m, m^{\prime}\right)-d_{n}\left(m, m^{\prime}\right) \leq 2^{-(n-1)}$. Thus, for $n$ suffuciently large,

$$
O_{\epsilon, r}:=\left\{m \in \mathcal{P}(S M): d_{n}\left(m, m_{\epsilon}\right)<r\right\} \subset O .
$$

For each $\alpha=\left(\alpha_{1}, \ldots, \alpha_{n}\right) \in \mathbb{R}^{n}$ write $\|\alpha\|_{n}=\sum_{k=1}^{n} 2^{-k}\left|\alpha_{k}\right|$. Set $\alpha_{\epsilon}:=\left(\int g_{1} d m_{\epsilon}, \ldots, \int g_{n} d m_{\epsilon}\right)=m_{\epsilon}\left(g^{(n)}\right)$ and

$$
O_{n, r}:=\left\{\alpha \in \mathbb{R}^{n}:\left\|\alpha_{\epsilon}-\alpha\right\|_{n}<r\right\} .
$$

Then, $g^{(n)}\left(O_{\epsilon, r}\right)=O_{n, r} \cap g^{(n)}(\mathcal{P}(S M))$. From Theorem 2 (2) we get,

$$
\begin{aligned}
& \liminf _{T \rightarrow+\infty} \frac{1}{T} \log \frac{\int\left(\sum_{\gamma_{x y} \in G_{\delta, T}: \delta_{\gamma_{x y}} \in O} e^{\int_{\gamma_{x y}} F}\right) d x d y}{\int\left(\sum_{\gamma_{x y} \in G_{\delta, T}} e^{\int_{\gamma_{x y}} F}\right) d x d y} \\
\geq & \liminf _{T \rightarrow+\infty} \frac{1}{T} \log \frac{\int\left(\sum_{\gamma_{x y} \in G_{\delta, T}: \delta_{\gamma_{x y}} \in O_{\epsilon, r}} e^{\int_{\gamma_{x y}} F}\right) d x d y}{\int\left(\sum_{\gamma_{x y} \in G_{\delta, T}} e^{\int_{\gamma_{x y}} F}\right) d x d y} \\
= & \liminf _{T \rightarrow+\infty} \frac{1}{T} \log \frac{\int\left(\sum_{\gamma_{x y} \in G_{\delta, T}: \delta_{\gamma_{x y}}\left(g^{(n)}\right) \in O_{n, r}} e^{\int_{\gamma_{x y}} F}\right) d x d y}{\int\left(\sum_{\gamma_{x y} \in G_{\delta, T}} e^{\int_{\gamma_{x y}} F}\right) d x d y} \\
\geq & -J_{g}\left(O_{n, r}\right) \\
\geq & -J_{g}\left(\alpha_{\epsilon}\right) \geq-J\left(m_{\epsilon}\right) \geq-J(O)-\epsilon,
\end{aligned}
$$

for any $\epsilon>0$. This completes the proof of the main Theorem 1 .

\subsection{Proof of Theorem 3.}

Proof. We have to show that the weak limits of

$$
m_{\delta, T}:=\frac{\int\left(\sum_{\gamma_{x y} \in G_{\delta, T}} e^{\int_{\gamma_{x y}} F} \delta_{\gamma_{x y}}\right) d x d y}{\int\left(\sum_{\gamma_{x y} \in G_{\delta, T}} e^{\int_{\gamma_{x y}} F}\right) d x d y}
$$


are contained in $\mathcal{P}_{e}(F)$.

Let $V \subset \mathcal{P}(S M)$ be a convex open neighborhood of $\mathcal{P}_{e}(F)$ and $\epsilon>0$. We consider a finite open cover $\left(B_{i}(\epsilon)\right)_{i \leq N}$ of $\mathcal{P}_{e}(F)$ by balls of diameter $\epsilon$ all contained in $V$. Define the measures on $S M$,

$$
m_{T, V}:=\frac{\int\left(\sum_{\gamma_{x y} \in G_{\delta, T}: \delta_{\gamma_{x y} \in V}} e^{\int_{\gamma_{x y}} F} \delta_{\gamma_{x y}}\right) d x d y}{\int\left(\sum_{\gamma_{x y} \in G_{\delta, T}} e^{\int_{\gamma_{x y}} F}\right) d x d y} .
$$

Decompose the set $U:=\cup_{i=1}^{N} B_{i}(\epsilon)$ in a disjoint union as follows,

$$
U=\cup_{j=1}^{N^{\prime}} U_{j}^{\epsilon},
$$

where the sets $U_{j}^{\epsilon}$ are disjoints and contained in one of the balls $\left(B_{i}(\epsilon)\right)_{i \leq N}$. We have

$$
\mathcal{P}_{e}(F) \subset U \subset V .
$$

We fix in each $U_{j}^{\epsilon}$ a probability measure $m_{j}, j \leq N^{\prime}$, and let $m_{0}$ be a probability measure distinct from the above ones (for example take $\left.m_{0} \in V \backslash U\right)$.

Set as usual,

$$
\nu_{T}(E):=\frac{\int\left(\sum_{\gamma_{x y} \in G_{\delta, T}: \delta_{\gamma_{x y}} \in E} e^{\int_{\gamma_{x y}} F}\right) d x d y}{\int\left(\sum_{\gamma_{x y} \in G_{\delta, T}} e^{\int_{\gamma_{x y}} F}\right) d x d y},
$$

and define,

$$
\beta_{T}=\sum_{j=1}^{N^{\prime}} \nu_{T}\left(U_{j}^{\epsilon}\right) m_{j}+\left(1-\nu_{T}(U)\right) m_{0} .
$$

We have $\sum_{j=1}^{N^{\prime}} \nu_{T}\left(U_{j}^{\epsilon}\right)=\nu_{T}(U)$. The probability measure $\beta_{T}$ lies in $V$ since it is a convex combination of elements in the convex set $V$. We have then $d\left(m_{\delta, T}, V\right) \leq d\left(m_{\delta, T}, \beta_{T}\right)$. We will show that

$$
d\left(m_{\delta, T}, \beta_{T}\right) \leq \epsilon \nu_{T}(U)+\frac{3}{2} \nu_{T}\left(U^{c}\right)
$$

where $U^{c}=\mathcal{P}(S M) \backslash U$ which is closed.

By definition of $m_{\delta, T}$ and $m_{T, V}$ and the fact that $U \subset V$,

$$
\sum_{k \geq 1} 2^{-k}\left|m_{\delta, T}\left(g_{k}\right)-m_{T, V}\left(g_{k}\right)\right| \leq \frac{1}{2} \nu_{T}\left(U^{c}\right) .
$$

It remains to show that $d\left(m_{T, V}, \beta_{T}\right) \leq \epsilon \nu_{T}(U)+\nu_{T}\left(U^{c}\right)$. We have for all $k \geq 1$,

$$
\left|m_{T, V}\left(g_{k}\right)-\beta_{T}\left(g_{k}\right)\right| \leq A+B+C
$$


where,

$$
\begin{aligned}
& A=\frac{\sum_{j=1}^{N^{\prime}} \int\left(\sum_{\gamma_{x y} \in G_{\delta, T}: \delta_{\gamma_{x y} \in U_{j}^{\epsilon}}} e^{\int_{\gamma_{x y}} F}\left|\delta_{\gamma_{x y}}\left(g_{k}\right)-m_{j}\left(g_{k}\right)\right|\right) d x d y}{\int\left(\sum_{\gamma_{x y} \in G_{\delta, T}} e^{\int_{\gamma_{x y}} F}\right) d x d y}, \\
& B=\frac{\int\left(\sum_{\gamma_{x y} \in G_{\delta, T}: \delta_{\gamma_{x y} \in V \backslash U}} e^{\int_{\gamma_{x y} F}} \delta_{\gamma_{x y}}\left(g_{k}\right)\right) d x d y}{\int\left(\sum_{\gamma_{x y} \in G_{\delta, T}} e^{\int_{\gamma_{x y}} F}\right) d x d y} \\
& C=\left|\left(1-\nu_{T}(U)\right) m_{0}\left(g_{k}\right)\right| .
\end{aligned}
$$

Thus, since we have for all $k \geq 1,\left\|g_{k}\right\|=1$, by definition of $\nu_{T}(15)$ we get,

$$
\begin{aligned}
& \sum_{k \geq 1} 2^{-k}\left|m_{T, V}\left(g_{k}\right)-\beta_{T}\left(g_{k}\right)\right| \\
\leq & \epsilon \sum_{j=1}^{N^{\prime}} \nu_{T}\left(U_{j}^{\epsilon}\right)+\frac{1}{2} \nu_{T}\left(U^{c}\right)+\frac{1}{2}\left(1-\nu_{T}(U)\right) \\
= & \epsilon \nu_{T}(U)+\nu_{T}\left(U^{c}\right) .
\end{aligned}
$$

Finally we have obtained that

$$
d\left(m_{\delta, T}, \beta_{T}\right) \leq \epsilon \nu_{T}(U)+\frac{3}{2} \nu_{T}\left(U^{c}\right) .
$$

This implies the desired inequality,

$$
d\left(m_{\delta, T}, V\right) \leq \epsilon \nu_{T}(U)+\frac{3}{2} \nu_{T}\left(U^{c}\right) .
$$

By Corollary 1, since $U^{c}$ is closed, we know that $\lim _{T \rightarrow \infty} \nu_{T}(U)=1$. Thus, $\lim \sup _{T \rightarrow \infty} d\left(m_{\delta, T}, V\right) \leq \epsilon$, for all $\epsilon>0$. We conclude that $\lim \sup _{T \rightarrow \infty} d\left(m_{\delta, T}, V\right)=0$. The neighborhood $V$ of $\mathcal{P}_{e}(F)$ being arbitrary, this implies that all limit measures of $m_{\delta, T}$ are contained in $\mathcal{P}_{e}(F)$. In particular, if $\mathcal{P}_{e}(F)$ is reduced to one measure $\mu$, this shows that $m_{T}$ converges to $\mu$.

\subsection{Proof of Theorem 5.}

\subsubsection{Proof of Part 1.}

Proof. Set for any $E \subset \mathcal{P}(S M)$,

$$
\nu_{T}(E)=\frac{\int_{M \times M}\left(\sum_{\gamma_{x y} \in G_{T}: \delta_{\gamma_{x y}} \in E} e^{\int_{\gamma_{x y}} F}\right) d x d y}{\int_{M \times M}\left(\sum_{\gamma_{x y} \in G_{T}} e^{\int_{\gamma_{x y}} F}\right) d x d y} .
$$


Let $\epsilon>0$. There exists a finite number of continuous functions $\omega_{1}, \cdots, \omega_{l}$ such that $K \subset \cup_{i=1}^{l} K_{i}$, where

$$
K_{i}=\left\{m \in \mathcal{P}(S M): \int \omega_{i} d m-Q\left(\omega_{i}\right)>J(K)-\epsilon\right\} .
$$

We can suppose that all the $\omega_{i}$ 's are non negative since adding a constant $c>0$ we have,

$$
\int\left(\omega_{i}+c\right) d m-Q\left(\omega_{i}+c\right)=\int \omega_{i} d m-Q\left(\omega_{i}\right) .
$$

Put

$$
\Gamma_{i}(x, y, T):=\left\{\gamma_{x y} \in G_{T}: \delta_{\gamma_{x y}} \in K_{i}\right\}
$$

and

$$
Z_{i}(T):=\int \sum_{\gamma_{x y} \in \Gamma_{i}(x, y, T)} e^{\int_{\gamma_{x y}} F} d x d y .
$$

From the definition of $\Gamma_{i}(x, y, T)$ we get,

$$
Z_{i}(T) \leq \int \sum_{\gamma_{x y} \in \Gamma_{i}(x, y, T)} e^{\int_{\gamma_{x y}} F} e^{l\left(\gamma_{x y}\right)\left(\int \omega_{i} d \delta_{\gamma_{x y}}-Q\left(\omega_{i}\right)-(J(K)-\epsilon)\right)} d x d y
$$

We decompose the interval $[0, T]$ into subintervals $[T-(j+1) \delta, T-j \delta]$ and set

$$
\Gamma_{i j}(x, y, T):=\left\{\gamma_{x y}: T-(j+1) \delta<l\left(\gamma_{x y}\right) \leq T-j \delta, \delta_{\gamma_{x y}} \in K_{i}\right\} .
$$

Since the functions $\omega_{i}$ were supposed non negative, we have $Q\left(\omega_{i}\right) \geq 0$ and then $Q\left(\omega_{i}\right)+J(K) \geq 0$. Thus, from Theorem 9 (1) and for $T$ sufficiently large,

$$
\begin{aligned}
Z_{i}(T) & \leq \int \sum_{\gamma_{x y} \in \Gamma_{i}(x, y, T)} e^{\int_{\gamma_{x y}}\left(F+\omega_{i}\right)} e^{l\left(\gamma_{x y}\right)\left(-Q\left(\omega_{i}\right)-(J(K)-\epsilon)\right)} d x d y \\
& \leq \sum_{j} e^{(T-(j+1) \delta)\left(-Q\left(\omega_{i}\right)-(J(K)-\epsilon)\right)} \int \sum_{\gamma_{x y} \in \Gamma_{i j}(x, y, T)} e^{\int_{\gamma_{x y}}\left(F+\omega_{i}\right)} d x d y \\
& \leq \sum_{j} e^{(T-(j+1) \delta)\left(-Q\left(\omega_{i}\right)-(J(K)-\epsilon)\right)} e^{(T-j \delta)\left(P\left(F+\omega_{i}\right)+\epsilon\right)} \\
& =\sum_{j} e^{(T-j \delta)(P(F)-J(K)+2 \epsilon)} e^{-\delta\left(-Q\left(\omega_{i}\right)-(J(K)-\epsilon)\right)} .
\end{aligned}
$$

We assumed that $J(K)<P(F)$, then $C:=\sum_{j} e^{-j \delta(P(F)-J(K)+2 \epsilon)}<\infty$. Setting $\lambda_{i}:=e^{-\delta\left(-Q\left(\omega_{i}\right)-(J(K)-\epsilon)\right)}$, we get for $T$ sufficiently large and 
Theorem $9(2)(P(F)>0)$,

$$
\begin{aligned}
\nu_{T}(K) & \leq \frac{\sum_{i=1}^{l} Z_{i}(x, y, T)}{\int \sum_{\gamma_{x y} \in G_{T}} e^{\int_{\gamma_{x y}} F} d x d y} \\
& \leq \sum_{i=1}^{l} \sum_{j} e^{T(-J(K)+3 \epsilon)} \lambda_{i} e^{-j \delta(P(F)-J(K)+2 \epsilon)} \\
& =e^{T(-J(K)+3 \epsilon)} C \sum_{i=1}^{l} \lambda_{i} .
\end{aligned}
$$

Take the logarithme, divide by $T$ and take the limsup,

$$
\limsup _{T \rightarrow \infty} \frac{1}{T} \log \nu_{T}(K) \leq-J(K)+3 \epsilon
$$

$\epsilon$ being arbitrary, this proves Theorem 5 (1).

\subsubsection{Proof of Part 2.}

Proof. As for the proof of part (2) of Theorem 1, the proof will be a consequence of the contraction principle,

$$
\liminf _{T \rightarrow \infty} \frac{\int\left(\sum_{\gamma_{x y} \in G_{T}: \delta_{\gamma_{x y}}(g) \in O_{n}} e^{\int_{\gamma x y} F}\right) d x d y}{\int\left(\sum_{\gamma_{x y} \in G_{T}} e^{\int_{\gamma_{x y}} F}\right) d x d y} \geq-J_{g}\left(O_{n}\right) .
$$

We follow the lines of the proof of Theorem 2 (2) with the same notations. Let $\delta>0$. We have,

$$
\frac{\int\left(\sum_{\gamma_{x y} \in G_{T}: \delta_{\gamma_{x y}}(g) \in O_{n}} e^{\int_{\gamma_{x y}} F}\right) d x d y}{\int\left(\sum_{\gamma_{x y} \in G_{T}} e^{\int_{\gamma_{x y}} F}\right) d x d y} \geq \frac{\int\left(\sum_{\gamma_{x y} \in \Gamma_{T}\left(O_{n}\right)} e^{\int_{\gamma_{x y}} F}\right) d x d y}{\int\left(\sum_{\gamma_{x y} \in G_{T}} e^{\int_{\gamma_{x y}} F}\right) d x d y}
$$

where,

$$
\Gamma_{T}\left(O_{n}\right):=\left\{\gamma_{x y} \in G_{\delta, T}: \delta_{\gamma_{x y}}(g) \in O_{n}\right\}
$$

Set

$$
Z_{T}\left(O_{n}\right):=\frac{\int\left(\sum_{\gamma_{x y} \in \Gamma_{T}\left(O_{n}\right)} e^{\int_{\gamma_{x y}} F}\right) d x d y}{\int\left(\sum_{\gamma_{x y} \in G_{T}} e^{\int_{\gamma_{x y}} F}\right) d x d y}
$$


Then $Z_{T}\left(O_{n}\right) \geq Z_{T}\left(O_{n, r}\right)$ and $($ see $(13))$,

$$
\begin{aligned}
& \frac{1}{T} \log Z_{T}\left(O_{n}\right) \\
\geq & \frac{1}{T} \log Z_{T}\left(O_{n, r}\right) \\
\geq & -r\left\|\beta_{\varepsilon}\right\|-\frac{\delta\left\|\beta_{\varepsilon} \cdot g\right\|_{\infty}}{T}+\left(Z_{T}\left(\beta_{\varepsilon} \cdot g\right)-\beta_{\varepsilon} \cdot \alpha_{\varepsilon}\right)+\frac{1}{T} \log Z_{T}^{\varepsilon}\left(O_{n, r}\right),
\end{aligned}
$$

where we have set,

$$
Z_{T}^{\varepsilon}\left(O_{n, r}\right):=\frac{\int\left(\sum_{\gamma_{x y} \in \Gamma_{T}\left(O_{n, r}\right)} e^{\int_{\gamma_{x y}}\left(F+\beta_{\varepsilon} \cdot g\right)}\right) d x d y}{\int\left(\sum_{\gamma_{x y} \in G_{\delta, T}} e^{\int_{\gamma_{x y}}\left(F+\beta_{\varepsilon} \cdot g\right)}\right) d x d y},
$$

and

$$
Z_{T}\left(\beta_{\varepsilon} \cdot g\right):=\frac{1}{T} \log \frac{\int\left(\sum_{\gamma_{x y} \in G_{\delta, T}} e^{\int_{\gamma_{x y}}\left(F+\beta_{\varepsilon} \cdot g\right)}\right) d x d y}{\int \sum_{\gamma_{x y} \in G_{T}} e^{\int_{\gamma_{x y}} F} d x d y} .
$$

From Theorem 9 (1) and (2) respectively,

$$
\lim _{T \rightarrow \infty} Z_{T}\left(\beta_{\varepsilon} \cdot g\right)=P\left(F+\beta_{\varepsilon} \cdot g\right)-P(F)=Q\left(\beta_{\varepsilon} \cdot g\right) .
$$

We proved in (14) that $\lim _{T \rightarrow \infty} Z_{T}^{\varepsilon}\left(O_{n, r}\right)=1$. Thus,

$$
\begin{aligned}
& \liminf _{T \rightarrow \infty} \frac{1}{T} \log \frac{\int\left(\sum_{\gamma_{x y} \in G_{T}: \delta_{\gamma_{x y}}(g) \in O_{n}} e^{\int_{\gamma_{x y}} F}\right) d x d y}{\int\left(\sum_{\gamma_{x y} \in G_{T}} e^{\int_{\gamma_{x y}} F}\right) d x d y} \\
\geq & \liminf _{T \rightarrow \infty} \frac{1}{T} \log \frac{\int\left(\sum_{\gamma_{x y} \in G_{\delta, T}: \delta_{\gamma_{x y}}(g) \in O_{n}} e^{\int_{\gamma_{x y}} F}\right) d x d y}{\int\left(\sum_{\gamma_{x y} \in G_{T}} e^{\int_{\gamma_{x y}} F}\right) d x d y} \\
\geq & -r\left\|\beta_{\varepsilon}\right\|+\left(Q\left(\beta_{\varepsilon} \cdot g\right)-\beta_{\varepsilon} \cdot \alpha_{\varepsilon}\right)+\lim _{T \rightarrow \infty} \frac{1}{T} \log Z_{T}^{\varepsilon}\left(O_{n, r}\right) \\
= & -r\left\|\beta_{\varepsilon}\right\|-I_{g}\left(\alpha_{\varepsilon}\right) \\
\geq & -r\left\|\beta_{\varepsilon}\right\|-J_{g}\left(O_{n}\right)-\varepsilon,
\end{aligned}
$$

for any $\varepsilon>0$. Since $r>0$ was arbitray choosen, we let $r \rightarrow 0$ and $\varepsilon \rightarrow 0$ respectively, this completes the proof of the contraction principle.

\subsection{Proof of Theorem 4.}

Proof. We have to prove that the weak limits of

$$
m_{T}:=\frac{\int\left(\sum_{\gamma_{x y} \in G_{T}} e^{\int_{\gamma_{x y}} F} \delta_{\gamma_{x y}}\right) d x d y}{\int\left(\sum_{\gamma_{x y} \in G_{T}} e^{\int_{\gamma_{x y}} F}\right) d x d y}
$$


are in $\mathcal{P}_{e}(F)$. For this and in order to follow the proof of Theorem 3 , we must show that we are able to apply Theorem 5 (1) to any open and convex neighborhood $V$ of $\mathcal{P}_{e}(F), V \subset \mathcal{P}(S M)$. There are minor changes due to the conditions $P(F)>0$ and $J(K)<P(F)$ in Theorem $5(1)$. Let $\nu \in \mathcal{P}_{e}(F)$ and $\mu$ a probability measure which is not invariant, i.e a non invariant element in $\mathcal{P}(S M) \backslash \mathcal{P}_{e}(F)$. We have $J(\nu)=0$ and $J(\mu)>0$ (by Remark 1). Consider a convex sum of these two measures, $m=\alpha \mu+\beta \nu$, where $\alpha+\beta=1(\alpha \neq 0$ and $\beta \neq 0)$. Observe that $m \notin \mathcal{P}_{e}(F)$. Indeed, assume that $m$ lies in $\mathcal{P}_{e}(F)$. In particular it is then invariant. But then, since $\nu$ is invariant by assumption, for all $t$ we will have

$$
\alpha \mu+\beta \nu=\alpha \mu \circ \phi_{t}+\beta \nu \circ \phi_{t}=\alpha \mu \circ \phi_{t}+\beta \nu .
$$

Thus $\mu=\mu \circ \phi_{t}$ for all $t$, which is a contradiction.

From the convexity of $J$ we get, $J(m) \leq \alpha J(\mu)$. Let $V \subset \mathcal{P}(S M)$ be an open and convex small neighborhood of $\mathcal{P}_{e}(F)$ such that $m \in V^{c}:=$ $K$ (note that in particular, for all other contained small neighborhood $V$ we will have $m \in V^{c}$ ). Therefore,

$$
\inf _{k \in K} J(k) \leq J(m) \leq \alpha J(\mu) .
$$

Now, since we have assumed that $P(F)>0$ then for a sufficiently small $\alpha>0$ we get from (17),

$$
J(K):=\inf _{k \in K} J(k) \leq P(F) .
$$

Thus $P(F)-J(K) \geq 0$. We can then apply Theorem 5 (1) to the closed sets $K=V^{c}$ and conclude with the proof of Theorem 3 .

\subsection{Proof of Theorem 6 .}

\subsubsection{Proof of Part (1).}

Proof. It is essentially the proof of Theorem 1 (1) with the following modifications since it is in part based on Theorem 8 and the following lemma (see [14] Lemma 4.3, [19] Lemma 3.33 p68).

Lemma 2. Let $(X, \mathcal{A}, \mu)$ be a probability space, and $f_{n}: X \rightarrow(0,+\infty)$ a sequence of integrable functions. Then for $\mu$ a.e $x \in X$

$$
\limsup _{n \rightarrow \infty} \frac{1}{n} \log f_{n}(x) \leq \limsup _{n \rightarrow \infty} \frac{1}{n} \log \int_{X} f_{n} d \mu .
$$


Set $F=0$ and proceed as in the proof of Theorem 1 (1) with the same notations. We have $Q(\omega)=P(\omega)-h_{\text {top }}$ and,

$$
\begin{aligned}
Z_{i}(x, y, T) & \leq \sum_{\gamma_{x y} \in \Gamma_{i}(x, y, T)} e^{\int_{\gamma_{x y}} \omega_{i}} e^{l\left(\gamma_{x y}\right)\left(-Q\left(\omega_{i}\right)-(J(K)-\epsilon)\right)} \\
& \leq C e^{T\left(-Q\left(\omega_{i}\right)-(J(K)-\epsilon)\right)} \sum_{\gamma_{x y} \in \Gamma_{i}(x, y, T)} e^{\int_{\gamma_{x y}} \omega_{i}}
\end{aligned}
$$

We have

$$
\nu_{T}(K) \leq \sum_{i=1}^{l} \frac{Z_{i}(x, y, T)}{\# G_{\delta, T}} .
$$

For all $(x, y)$ and $T$ sufficiently large (depending on $(x, y)$ ), it follows from Theorem 8 (2) that,

$$
\# G_{\delta, T} \geq e^{T\left(h_{t o p}-\epsilon\right)} .
$$

On the other hand it follows from Lemma 2 above (which can be applied to continuous time) and Theorem 9 (1),

$$
\begin{aligned}
& \limsup _{T \rightarrow \infty} \frac{1}{T} \log \sum_{\gamma_{x y} \in \Gamma_{i}(x, y, T)} e^{\int_{\gamma_{x y}} \omega_{i}} \\
\leq & \limsup _{T \rightarrow \infty} \frac{1}{T} \log \sum_{\gamma_{x y} \in G_{\delta, T}} e^{\int_{\gamma_{x y}} \omega_{i}} \\
\leq & \limsup _{T \rightarrow \infty} \frac{1}{T} \log \int\left(\sum_{\gamma_{x y} \in G_{\delta, T}} e^{\int_{\gamma_{x y}} \omega_{i}}\right) d x d y \\
\leq & P\left(\omega_{i}\right),
\end{aligned}
$$

for $(x, y)$ in a subset $B_{i}$ of $M \times M$ of full Lebesgue measure and $i \leq l$. The set $B_{i}$ can be taken independent from $i$ for evident reasons, and then we will say that the above inequalities hold for a.e $(x, y)$ in a set $B$. However, note that $B$ depends on the set of functions $\left\{\omega_{i}, i \leq l\right\}$ which means that $B$ depends on $K$. The proof can be now achieved similarily : let $(x, y) \in B$ fixed and $T$ sufficiently large (depending on $(x, y))$,

$$
\begin{aligned}
\nu_{T}(K) & \leq \sum_{i=1}^{l} \frac{Z_{i}(x, y, T)}{\# G_{\delta, T}} \\
& \leq C \sum_{i=1}^{l} e^{T\left(P\left(\omega_{i}\right)+\epsilon\right)} e^{-T\left(h_{t o p}-\epsilon\right)} e^{T\left(-Q\left(\omega_{i}\right)-(J(K)-\epsilon)\right.} \\
& =C l e^{T(-J(K)+3 \epsilon)} .
\end{aligned}
$$


Thus $\lim \sup _{T \rightarrow \infty} \frac{1}{T} \log \nu_{T}(K) \leq-J(K)$.

\subsubsection{Proof of Part (2).}

Proof. Set

and

$$
\nu_{T}(E)=\frac{\#\left\{\gamma_{x y} \in G_{\delta, T}: \delta_{\gamma_{x y}} \in E\right\}}{\# G_{\delta, T}}
$$

$$
\mu_{T, V}(x, y):=\frac{\sum_{\gamma_{x y} \in G_{\delta, T}: \delta_{\gamma_{x y}} \in V} \delta_{\gamma_{x y}}}{\# G_{\delta, T}} .
$$

Thus, proceeding as in the proof of Theorem 3, we get for any open and convex neighborhood $V$ of $\mathcal{P}_{e}(0)$ and all $(x, y)$,

$$
d\left(\mu_{T}(x, y), V\right) \leq \epsilon \nu_{T}(U)+\frac{3}{2} \nu_{T}\left(U^{c}\right) .
$$

Let $\left(V_{i}\right)_{i \geq 1}$ be a decreasing sequence of sets of the type $V$ such that $\cap_{i \geq 1} V_{i}=\mathcal{P}_{e}(0)$. For $(x, y)$ in a set $B_{V_{i}}$ of full Lebesgue measure we get from Theorem 6 (1) and (18),

$$
\limsup _{T \rightarrow \infty} d\left(\mu_{T}(x, y), V_{i}\right) \leq \epsilon
$$

for all $\epsilon>0$. Thus $\lim \sup _{T \rightarrow \infty} d\left(\mu_{T}(x, y), V_{i}\right)=0$. Therefore, if $\mu(x, y)$ is a weak limit of $\mu_{T}(x, y),(x, y) \in B_{V_{i}}$, we will have $d\left(\mu(x, y), V_{i}\right)=0$. Also, there exists a set $B$ of full Lebesgue measure where we have $d\left(\mu(x, y), V_{i}\right)=0$ for all $i \geq 1$. From this we deduce that $\mu(x, y) \in$ $\mathcal{P}_{e}(0)$ for $(x, y) \in B$.

\subsection{Proof of Theorem 7.}

\subsubsection{Proof of Part (1).}

Proof. The proof adapts the arguments of the proof of Theorem 5 (1). Set for any $E \subset \mathcal{P}(S M)$,

$$
\nu_{T}(E)=\frac{\#\left\{\gamma_{x y} \in G_{T}: \delta_{\gamma_{x y}} \in E\right\}}{\# G_{T}} .
$$

Recall that the functional $Q$ corresponding to the potential $F=0$ is given by $Q(\omega)=P(\omega)-h_{\text {top }}$.

Let $\epsilon>0$. There exists a finite number of continuous functions $\omega_{1}, \cdots, \omega_{l}$ such that $K \subset \cup_{i=1}^{l} K_{i}$, where

$$
K_{i}=\left\{m \in \mathcal{P}(S M): \int \omega_{i} d m-Q\left(\omega_{i}\right)>J(K)-\epsilon\right\} .
$$

Again we can suppose that all the $\omega_{i}$ 's are non negative.

Put

$$
\Gamma_{i}(x, y, T):=\left\{\gamma_{x y} \in G_{T}: \delta_{\gamma_{x y}} \in K_{i}\right\}
$$


and

$$
Z_{i}(x, y, T):=\# \Gamma_{i}(x, y, T) .
$$

From the definition of $\Gamma_{i}(x, y, T)$ we get,

$$
Z_{i}(x, y, T) \leq \sum_{\gamma_{x y} \in \Gamma_{i}(x, y, T)} e^{l\left(\gamma_{x y}\right)\left(\int \omega_{i} d \delta_{\gamma_{x y}}-Q\left(\omega_{i}\right)-(J(K)-\epsilon)\right)}
$$

Set

$$
\Gamma_{i j}(x, y, T):=\left\{\gamma_{x y}: T-(j+1) \delta<l\left(\gamma_{x y}\right) \leq T-j \delta, \delta_{\gamma_{x y}} \in K_{i}\right\} .
$$

Since the functions $\omega_{i}$ were supposed non negative, we have $Q\left(\omega_{i}\right) \geq 0$ and then $Q\left(\omega_{i}\right)+J(K) \geq 0$. Thus, from Theorem 9 (1) and for $T$ sufficiently large,

$$
\begin{aligned}
Z_{i}(x, y, T) & \leq \sum_{\gamma_{x y} \in \Gamma_{i}(x, y, T)} e^{\int_{\gamma_{x y}} \omega_{i}} e^{l\left(\gamma_{x y}\right)\left(-Q\left(\omega_{i}\right)-(J(K)-\epsilon)\right)} \\
& \leq \sum_{j} e^{(T-(j+1) \delta)\left(-Q\left(\omega_{i}\right)-(J(K)-\epsilon)\right)} \sum_{\gamma_{x y} \in \Gamma_{i j}(x, y, T)} e^{\int_{\gamma_{x y}} \omega_{i}} \\
& \leq \sum_{j} e^{(T-(j+1) \delta)\left(-Q\left(\omega_{i}\right)-(J(K)-\epsilon)\right)} e^{(T-j \delta)\left(P\left(\omega_{i}\right)+\epsilon\right)} \\
& =\sum_{j} e^{(T-j \delta)\left(h_{t o p}-J(K)+2 \epsilon\right)} e^{-\delta\left(-Q\left(\omega_{i}\right)-(J(K)-\epsilon)\right)} .
\end{aligned}
$$

We assumed that $J(K)<h_{\text {top }}$, then $C:=\sum_{j} e^{-j \delta\left(h_{t o p}-J(K)+2 \epsilon\right)}<\infty$. Setting $\lambda_{i}:=e^{-\delta\left(-Q\left(\omega_{i}\right)-(J(K)-\epsilon)\right)}$, we get for $T$ sufficiently large and Theorem 8 (2),

$$
\begin{aligned}
\nu_{T}(K) & \leq \sum_{i=1}^{l} \frac{Z_{i}(x, y, T)}{\# G_{T}} \\
& \leq \sum_{i=1}^{l} \sum_{j} e^{T(-J(K)+3 \epsilon)} \lambda_{i} e^{-j \delta\left(h_{t o p}-J(K)+2 \epsilon\right)} \\
& =e^{T(-J(K)+3 \epsilon)} C \sum_{i=1}^{l} \lambda_{i} .
\end{aligned}
$$

Take the logarithme, divide by $T$ and take the limsup,

$$
\limsup _{T \rightarrow \infty} \frac{1}{T} \log \nu_{T}(K) \leq-J(K)+3 \epsilon .
$$

$\epsilon$ being arbitrary, this proves Theorem 7 (1).

4.10.2. Proof of Part (2). The proof follows readily from the proof of Theorem 6 (2) and Theorem 7 (1). 


\section{REFERENCES}

[1] Amroun A. On the measures of maximal entropy for the geodesic flow. Expo. Math. 23, 151-160, 2005.

[2] Amroun A. Equilibrium states for the geodesic flows. Math Zeitschrift. 252, 797-810, 2006.

[3] Berger M, Bott R. Sur les variétés a courbure strictement positive. Topology, Vol 1, 1962.

[4] Burns K. Gutkin E. Growth of the number of geodesics between points and insecurity for Riemannian manifolds. Dis Cont Dyn Sys, vol 21, N2, 2008.

[5] Bowen R. The equidistribution of closed geodesics. Amer. J. Math. 94, 413-423, 1972.

[6] Bowen R. Periodic orbits for hyperbolic flows. Amer. J. Math. 94, 1-30, 1972.

[7] Chengbo Yue. Rigidity and dynamics around manifolds of negative curvature. Math Res Letters 1, 123-147, 1994.

[8] Chengbo Yue. The ergodic theory of discrete isometry groups on manifolds of variable negative curvature. Trans Ame Math Soc, Vol 348, Numb 12, 4965$5005,1996$.

[9] Dembo A. Zeitouni O. Large deviations techniques and Applications. Springer, Application of Mathematics, vol 38, 1998.

[10] Federer H. Geometric measure theory. Springer, New York, 1969.

[11] Kifer Y. Large deviations in dynamical systems stochastic processes. Trans. Amer. Math. Soc. 321, 505-525, 1988.

[12] Knieper G. The uniquesness of the measure of maximal entropy for geodesic flow on rank 1 manifolds.

[13] Kozlovski O S. An integral formula for topological entropy of $C^{\infty}$ maps. Ergodic Theory Dynam Systems. 18, 405-424, 1998.

[14] Mañé R. On the topological entropy of the geodesic flows. J Diff Geom 45, 74-93, 1989.

[15] Morgan F. Geometric measure theory. Academic Press 1988.

[16] Newhouse S. Continuity properties of entropy. Ann. Math. 129, 215-235, 1989.

[17] Parry W. Equilibrium states and weighted uniform distribution of closed orbits, Dynamical Systems. LNM vol 1342, Springer, Berlin, 1988.

[18] Paternain G. P. Topological pressure for geodesic flows. Ann. Scient. Éc. Norm. Sup. 4eme série, t 33, 121-138, 2000.

[19] Paternain G. P. Geodesic flows. Progress in mathematics, 180, Birkhauser, 2000.

[20] Pollicott M. Closed geodesic distribution for manifolds of nonpositive curvature. Disc. Cont. Dyn. Syst. Vol 2, Num 2, 1996.

[21] Pollicott M. Large deviations, Gibbs measures and closed orbits for hyperbolic flows. Math. Z. 220, 219-230, 1995.

[22] Przytycki F. An upper estimation for topological entropy of diffeomorphisms. Invent Math. 59, 205-213, 1980.

[23] Rockafellar R T. Convex analysis. Princeton Univ Press, Princeton N J 1970.

[24] Ruelle D. Thermodynamical formalism. Encyclopedia of Math and its Appl, vol 5, Addison-Wesley, Reading, Mass, 1978.

[25] Walters P. An introduction to ergodic theory. Graduate Text in Mathematics 79, Springer, Berlin 1982. 